Revue d'histoire de l'Amérique française

REVUE D.HISTOIRE DE L'AMÉRIQUE FRANÇAISE

\title{
L’immigration française en Louisiane, 1718-1721
}

\section{Mathé Allain}

Volume 28, numéro 4, mars 1975

URI : https://id.erudit.org/iderudit/303395ar

DOI : https://doi.org/10.7202/303395ar

Aller au sommaire du numéro

Éditeur(s)

Institut d'histoire de l'Amérique française

ISSN

0035-2357 (imprimé)

1492-1383 (numérique)

Découvrir la revue

Citer cette note

Allain, M. (1975). L'immigration française en Louisiane, 1718-1721. Revue

d'histoire de l'Amérique française, 28(4), 555-564.

https://doi.org/10.7202/303395ar d'utilisation que vous pouvez consulter en ligne.

https://apropos.erudit.org/fr/usagers/politique-dutilisation/ 


\title{
NOTES DE RECHERCHE
}

I

\section{L'IMMIGRATION FRANÇAISE EN LOUISIANE 1718-1721*}

\author{
Mathé Allain \\ University of Southwestern \\ Louisiana \\ Lafayette, Louisiana
}

A la mort de Louis XIV, il n'y avait guère plus de 215 habitants, y compris les effectifs militaires, dans le vaste territoire de la Louisiane. ${ }^{1}$ Les progrès avaient donc été très lents depuis la fondation de la colonie en 1699 par le Canadien Pierre Le Moyne, sieur d'Iberville. Il n'est guère difficile de trouver les raisons de cette lenteur. Fondée pour arrêter l'expansion espagnole de la Floride et du Texas dans la vallée du Mississipi et pour décourager les incursions anglaises, la Louisiane, lointaine et coûteuse, n'avait guère de valeur par elle-même. L'intendant de la Nouvelle-France, Jean Talon, avait bien répété depuis 1665 que le roi de France devait conquérir l'intérieur du continent et expulser les Espagnols de la vallée. ${ }^{2}$ Et le rêve de richesses minières, comparables à celles des colonies espagnoles, donnait une certaine impulsion à la colonisation. Mais les guerres européennes, surtout la guerre si meurtrière et coûteuse de la Succession d'Espagne, ne laissèrent à la couronne française ni les hommes ni les fonds nécessaires pour les aventures coloniales. Une autre conséquence de la guerre fut l'accumulation d'énormes dettes; aussi en 1712, l'idée était-elle née d'utiliser ce poids mort que représentait la Louisiane pour satisfaire un des plus gros créanciers de la couronne. Le roi offrit donc le monopole du commerce louisianais à Antoine Crozat qui l'accepta en escomptant d'énormes profits.

Les grandes espérances de Crozat et de la couronne furent assez vite déçues et la Louisiane demeura le poids mort qu'elle

\footnotetext{
* Communication présentée au Congrès de l'IHAF, le 26 octobre 1974. 255.

1 Marcel Giraud, Histoire de la Louisiane française (Paris, 1963), I:

2 John Anthony Caruso, The Mississippi Valley Frontier: The Age of French Exploration (Indianapolis, 1966), 149.
} 
était avant le monopole du financier. Le marasme économique qui régnait en France après la guerre de la Succession d'Espagne est principalement responsable du manque d'essor de la colonie. Mais de plus, la colonie ne présentait aucune possibilité de développement économique aussi longtemps que sa population demeurerait aussi peu nombreuse - en 1712, au moment de l'établissement du monopole de Crozat, D'Artaguiette parlait de 27 familles $;^{3}$ or, les lettres patentes de Crozat n'exigeaient de lui que l'établissement annuel de 10 hommes et de 10 femmes dans la colonie. Crozat savait bien qu'un tel apport était insuffisant, mais, selon lui, c'était au souverain qu'il incombait de peupler la colonie. ${ }^{4}$ En 1713, après la paix d'Utrecht, les circonstances auraient été assez propices à l'immigration car la misère créait, provisoirement, un climat favorable. Mais il aurait fallu agir immédiatement. Le léger rétablissement économique de 1714 suffit à décourager ces velléités d'émigrations volontaires. Or, le roi, aussi bien que le comte de Pontchartrain, ministre de la Marine, était inexorablement opposé à l'émigration forcée.

L'émigration volontaire soulevait plusieurs problèmes. Il y avait d'abord la mauvaise réputation de la colonie. Avant l'avalanche de pamphlets et de mémoires de 1717 , on ignorait à peu près tout sur la Louisiane en France (en dehors des milieux savants, cf. Giraud, II : 12-26) et le peu qu'on en savait était défavorable. La peinture que l'on faisait de la colonie du Mississipi était si peu attrayante que les filles à marier en partance pour la Louisiane en 1704 durent être surveillées de près pour éviter toute désertion. La situation ne s'améliora guère, malgré les études scientifiques et les écrits érudits, et, en 1719, Jean-Pierre Purry pouvait écrire: "C'est une chose certaine que le païs de la Louisiane est dans une très mauvaise réputation et qu'il y a très peu de gens dans le monde qui ne s'en fasse une idée des plus affreuses." La réputation de la pauvre colonie est si mauvaise, continue le Suisse, que "aux spectacles publics lorsqu'on représente un homme qui méritte la mort ou quelque autre genre de supplice équivalent, on le condamne d'être envoyé au Missipy; de sorte qu'il n'y a rien, sur le pied où les choses sont, de plus honteux dans le Monde pour un honnête homme, que d'aller en ce païs-là". ${ }^{5}$

De plus, le peuplement étant fortement handicapé par l'attitude du gouvernement, les justifications données pour l'explora-

${ }^{3} \mathrm{AC}$ C C13a, $2: 800$, D'Artaguiette à Pontchartrain, 8 sept. 1712.

4 AC, C13a, 3:378-379, Mémoire sur l'état présent de la colonie de la Louisiane, octobre 1713.

5 AC, C13a, 5:76, Mémoire, 1719. 
tion et l'établissement de la colonie n'étaient guère propices à la colonisation. La Salle avait exploré le Mississipi pour "établir", disait-il, "par ce moyen le commerce des Pelleteries de Castors et de Boeufs sauvages". ${ }^{6}$ Mais se rendant compte que cet argument ne suffirait à convaincre ni le roi ni Colbert, La Salle y ajouta un argument stratégique: le poste permettrait de "fatiguer les Espagnols dans le lieux d'où ils tirent toutes leurs richesses", 7 et de là les Français pourraient facilement conquérir les colonies espagnoles. Pour renforcer son argument, La Salle avait même ajouté à son mémoire une carte de la région où l'embouchure du Mississipi avait été sciemment placée à plus de mille kilomètres de son emplacement véritable. ${ }^{8}$

Après la mort de l'explorateur, son dévoué compagnon Henri de Tonti, qui tenait à "parachever" l'œuvre de La Salle, continua à présenter un argument stratégique: un établissement sur la côte empêcherait les Anglais de s'étendre sur le golfe du Mexique, servirait de base pour attaquer la Nouvelle-Espagne, et protégerait le Canada qui serait perdu si les Anglais s'établissaient dans la vallée du Mississipi. ${ }^{9}$

En 1698, lorsque d'Iberville prépare l'expédition au cours de laquelle il établira la colonie, les instructions qu'il reçoit de la couronne sont bien précises: il doit occuper l'embouchure du Mississipi et "empescher qu'aucune autre nation ne mette pied à terre aux environs de cette embouchure". Il n'est pas question de colonisation mais seulement d'établir un fort avec le minimum de garnison. ${ }^{10}$

C'est encore l'argument stratégique qui convainc le roi et le ministre de maintenir la France en Louisiane en 1707. Le roi, tenté d'abandonner la Louisiane, demande à être informé "de l'utilité dont cette colonie peut estre pour le Royaume" parce que si elle "estoit désavantageuse" il "prendrait le party d'abandonner cette colonie sans s'engager davantage"."11 Le commerce des castors, prôné par La Salle, n'est pas un argument en faveur du maintien de la présence française, car le roi interdisait cette traite en Louisiane "afin de pouvoir soutenir la colonie de Canada". ${ }^{12}$ Mais, ainsi que Pontchartrain le souligne, la Louisiane est

6 Paris. Cartes et Plans, vol. 64, no 4, I: 1682, Relation des découvertes et des voyages de la Salle 1679 à 1681.

7 AC, C13a, $64: 1684$.

8 Caruso, op. cit., 202.

9 Pierre Margry, Découvertes et Etablissements des Français... (Paris, 1880), 4.

$10 \mathrm{AC}, \mathrm{B} 20,111-113$, Instructions, 23 juillet 1698.

11 AC, B 29:278, A M. de Muy, 30 juin 1707.

12 Ibid. 
indispensable pour contrecarrer l'expansion anglaise et "c'est uniquement pour pouvoir s'y opposer, et l'arrester que le Roy fait la dépense de soutenir le poste de la Mobile". ${ }^{13}$

Crozat, bien entendu, espérait retirer de gros bénéfices de son monopole - Giraud l'appelle "âprement intéressé" ${ }^{14}$ - et attendait beaucoup des mines que Lamothe Cadillac lui dépeignait sous un jour des plus flatteurs. ${ }^{15}$ Mais s'étant rapidement rendu compte qu'il ne tirerait aucun profit d'une colonie faiblement peuplée et voulant convaincre la couronne d'assumer la tâche de peuplement, c'est à des arguments stratégiques que le financier fit appel. La Louisiane était indispensable pour défendre le $\mathrm{Ca}-$ nada, surtout après Utrecht: "Sy les anglois mettoient dans leurs interests toutes les nations qui habitent le haut de la Rivière de Mississipy, non seulement la communication avec le Canada se trouverait coupée, mais le Canada, qui n'est déjà pas trop en sûreté depuis la cession de l'Acadie et de Plaisance aux anglois, serait en bien plus grand danger." 16 Quelques mois plus tard il revient à la charge: "Si les anglois s'établissoient sur le fleuve St. Louis ... ils prendroient en peu d'années tout le Mexique... et... ils prendroient le Canada par les derrières." 17 Il faudrait donc que la France poursuive une politique de peuplement vigoureuse. Le climat d'opinion commence à changer, un autre mémoire écrit entre 1715 et 1716 donne autant d'importance aux possibilités d'agriculture de la Louisiane qu'à la défense des Canadiens qui, sans la Louisiane, risqueraient de se trouver "enfermez de tous costez et hors d'état de résister". 18

Même LaMothe Cadillac, ce gouverneur envoyé par Crozat qui ne trouvait rien de bon en Louisiane, - "la colonie ne vaut pas un fétu de paille à l'heure qu'il est", déclarait-il en 1713 admet son importance stratégique.19 "Rien ne peut incliner sa Majesté à conserver ce païs", pense-t-il, si ce n'est les perspectives assez aléatoires de commerce et de richesses minières, mais surtout la possibilité, en cas de rupture avec l'Espagne, que la colonie serve de base pour les corsaires et pour les expéditions contre les possessions espagnoles. ${ }^{20}$

${ }^{13}$ AC, B $30: 299$, A M. de la Ranette, 17 oct. 1708.

14 Giraud, I: 233.

15 AC, C13a, 2:678, La Mothe Cadillac, 20 juin 1712.

16 AC, Ba, 3:363-5, Mémoire de Crozat, 1713.

17 AC, C13a, 3:505, Mémoire de Crozat, 4 sept. 1714.

18 AC, C13a, 4:998, Mémoire sur l'établissement de la Louisiane (par Crozat ?) sans date (1716 ?).

19 AC, C13a, 3:47, A Pontchartrain, 26 oct. 1713.

20 AC, C13, 3:420-421, A Pontchartrain, 20 fév. 1714. 
La Louisiane était donc, en 1715 , un territoire éloigné et dépouillé de valeur propre. Cette colonie présentait surtout une importance stratégique pour la défense du Canada et l'attaque de la Nouvelle-Espagne. Il n'est donc pas surprenant que le peuplement de la colonie ait été négligé et que la population, déjà infime, fût principalement pour ne pas dire exclusivement composée de soldats ou d'aventuriers et non pas de paysans. ${ }^{21}$

Les premières années du monopole de Crozat n'avaient guère servi à consolider les débuts chancelants de l'établissement. En 1704 Nicolas de la Salle écrivait qu'il y avait en Louisiane 180 soldats, 27 familles avec 3 fillettes et 7 garçonnets, 6 jeunes Indiens et 5 Indiennes. ${ }^{22}$ Le dénombrement de 1706 donnait 24 familles avec 34 enfants, ${ }^{23}$ et quand La Salle prépara son recensement en 1708 , le nombre de familles était demeuré le même, mais celui des enfants avait diminué, 25 au lieu de $34 .^{24} \mathrm{En}$ 1708, LaSalle comptait en tout 279 personnes (dont 6 malades), y compris une garnison de 122 soldats et 80 esclaves indiens. ${ }^{25}$ En septembre 1712, au moment même où Crozat recevait ses lettres patentes, d'Artaguiette dénombrait 27 familles, un gain insignifiant. ${ }^{26}$ L'année suivante, Duclos y trouvait 35 familles, en dehors des gens au service du roi. ${ }^{27}$ Deux ans plus tard on y recensait 215 personnes dont 160 hommes de troupe. ${ }^{28} \mathrm{Au}$ lieu de progresser pendant les premières années du régime de Crozat, la Louisiane semble avoir légèrement régressé.

L'immigration volontaire n'avait certainement pas fait ses preuves. La mort de Louis XIV et l'avènement d'une nouvelle équipe, moins humanitaire et moins scrupuleuse, semblait devoir changer le sort de la colonie. On savait le régent, Philippe d'Orléans, intelligent et ouvert: l'intérêt que le comte de Toulouse, "personnalité dirigeante" 29 du Conseil de la Marine, portait aux questions coloniales était aussi bien connu. De fait un changement se fit immédiatement sentir dans le climat d'opi-

21 Pour un excellent résumé, voir Henri Gravier, La Colonisation de la Louisiane à l'époque de John Law, octobre 1717-janvier 1721 (Paris, 1909), 3-5.

22 AC, C13a, $1: 469$, La Salle, 31 août 1704.

23 Giraud, I: 154.

24 AC, C13a, 2:225-226, La Salle, 12 août 1708.

25 AC, C13a, 2:171-172, La Salle, 12 août 1708.

26 AC, C13a, 2:799-800, D'Artaguiette, 8 sept. 1712. D'Artaguiette fut ordonnateur du $30^{\circ}$ juin 1707 au 24 décembre 1712.

27 AC, C13a, 3:212, Duclos à Pontchartrain. Dubois Duclos fut ordonnateur de la Louisiane du 24 décembre 1712 au 12 novembre 1716.

$28 \mathrm{AC}, \mathrm{D} 2 \mathrm{c}, 51: 6-8$, Duclos, 31 août 1715 .

29 Giraud, I: 5. 
nion, changement que Giraud décrit en menus détails. ${ }^{30}$ Non seulement par l'attitude du Conseil de la Marine envers l'esclavage des Noirs et des Indiens, mais encore "par un nouveau démenti à ce respect de la liberté individuelle qu'il défend dans sa correspondance, il cède sur le principe de l'émigration forcée contre laquelle Pontchartrain s'est toujours élevé". ${ }^{31}$ Le Conseil adopte sans réserve le principe déjà suggéré par Crozat de l'émigration de faux-sauniers et de filles des hôpitaux. $^{32}$ Sur les instances des marchands de la Rochelle et de Nantes, le Conseil avait déjà décidé de faire transporter en Louisiane les vagabonds qui inquiétaient tant les villes. Finalement, la politique d'émigration forcée allait être appliquée aux déserteurs, aux fraudeurs de tabac, aux jeunes libertins, aux filles publiques, et même à des criminels. ${ }^{33}$

Aucune déportation n'eut lieu pendant la période de Crozat. La première émigration de faux-sauniers et de filles des hôpitaux qui aurait dû s'effectuer en mars 1717 fut retardée. En fait, ce groupe s'embarqua le 25 octobre 1717 sur le Neptune, la Dauphine et la Vigilante, arrivant dans la colonie en février de l'année suivante. ${ }^{34}$ Une fois la démission de Crozat acceptée par le Conseil de la Marine le 13 janvier 1717,35 la compagnie de John Law fut créée par lettres patentes en août de la même année. ${ }^{36}$

Les dernières années du monopole de Crozat furent caractérisées comme des années de transition. Du point de vue du peuplement, presque rien ne se fit: en 1712 il y avait 27 familles; en 1718, Charles Legac dénombre 350 à 400 personnes dans la colonie, y compris les hommes de troupe. ${ }^{37}$ Mais en partie sous l'influence du financier et des mémoires dont il inonde le Conseil, les attitudes changent et, au moment même où Crozat renonce à son monopole, le Conseil est prêt à encourager l'émigration par n'importe quel moyen.

30 Giraud, II: 27-37.

31 Giraud, II : 31.

32 AC, C13a, 4:71-73, Mémoire, 11 fév. 1716.

33 Sheila T. Sturdivant, "Rich Man, Poor Man, Beggar Man, Thief: Frenchmen Exiled to Louisiana, 1717-1721," unpublished thesis (USL, 1971), 19-20.

$34 \mathrm{AC}, \mathrm{C} 5 \mathrm{~b}, 37$.

35 AC, C13a, 5:4, Délibération du Conseil, 13 janvier 1717.

36 AC, B 45 bis: 51 , lettres patentes, août 1717 .

37 Glenn R. Conrad, Immigration and War: Louisiana, 1718-1721 from the Memoir of Charles Legac (Lafayette, 1970), 1. Charles Legac, un des premiers directeurs de la Compagnie d'Occident, arriva en Louisiane le 25 août 1718. Nommé directeur général de la Compagnie à l'Isle aux Vaisseaux, il demeura dans la colonie pendant toute la période de Law. 
L'avènement de Law fut d'importance cruciale dans l'histoire du peuplement de la Louisiane française. Quelles que soient les questions que l'on puisse soulever à l'égard du "système" et de la spéculation frénétique qu'il encouragea, il n'y a aucun doute que Law, en plaçant la Louisiane au cœur même de son système, donna à la colonie un essor démographique et économique dont elle ne connut plus d'égal pendant la période française. Pour la première fois la Louisiane bénéficia d'un effort concerté de peuplement, d'autant plus que l'effet de la politique gouvernementale de déportation est renforcé par l'immigration volontaire encouragée par une propagande effrénée.

L'apport d'immigrants est remarquable. En 1718 il arrive 741 personnes; en 1719, 1367 (et le premier arrivage de Noirs, 450 ); en 1720,2576 (et 127 Noirs); en 1721, 360 (et 868 Noirs) ${ }^{38}$ Parmi ces nouveaux arrivés, on compte, d'octobre 1717 à mai 1721, 1278 déportés, tant faux-sauniers et fraudeurs que vagabonds, "exilés" et "libertins" ${ }^{39}$; sur les 1215 femmes qui arrivent pendant la même période, 160 sont des prisonnières tirées de la Salpêtrière et d'autres hôpitaux. Cette politique d'émigration forcée est critiquable du point de vue moral: Blumenthal, par exemple, dit que "dans tout le peuplement de l'Amérique coloniale, aucune période n'égale en dégradation l'infâme époque de John Law". ${ }^{40}$ Mais elle mérite surtout d'être critiquée du point de vue pratique, car, malgré les chiffres assez impressionnants, elle représente un échec total.

En effet l'étude des listes de passagers révèle rapidement que les émigrants forcés ne firent pas souche en Louisiane. Les noms des faux-sauniers, des fraudeurs, des déserteurs, des vagabonds ont à jamais disparu. Les habitants ainsi forcés de s'exiler en Louisiane ou bien repassaient en France à la première occasion, ou, le plus souvent, mal préparés aux rigueurs d'un pays en voie de développement, mouraient de privations et de maladies. Les filles déportées ne fondèrent pas de foyers non plus. John Duffy insinue ironiquement que l'absence de progéniture chez ces femmes soulève des questions quant aux effets délétères du climat louisianais sur la fertilité humaine. ${ }^{41}$

38 Voir les listes de passagers dans Glenn R. Conrad, First Families of Louisiana (Baton Rouge, 1970), I: 1-140; Grady W. Kilman, "Slavery and Forced Labor in Colonial Louisiana, 1699 to 1803", thèse (USL, 1972), 48-51.

39 AC, C13c: 329-331, Mémoire, mai 1721. 1962), 98 .

40 Walter Hart Blumenthal, Brides from Bridewell (Rutland, Vt., 41 The Rudolf Matas History of Medicine in Louisiana (Baton Rouge, 1958), I: 24. 
$\mathrm{Au}$ cours de ces années arrivent en Louisiane de nombreux émigrants, français et alsaciens, dont les descendants formeront l'élite économique et sociale de la période française et même audelà : les de la Houssaye, de Laire, d'Hauterive, Pellerin, Broutin, Soileau, Olivier, Tournoir, Piquery, Courtableau. Viennent aussi les Schexnayder, Waguespack, Haydel, Himmel, et Frederic qui se révéleront d'excellents colons et qui s'assimileront si complètement à la population française que leurs descendants oublieront largement leur origine allemande. Mais ce n'est pas parmi les émigrants forcés qu'il faut les chercher. Ce sont les concessionnaires et leurs gens qui fondent ce que Glenn Conrad appelle "Les premières familles de Louisiane". Selon le mémoire anonyme de 1721, sous le régime de Law, vinrent en Louisiane 119 concessionnaires avec leurs gens et 2462 engagés - artisans et travailleurs - séduits, indubitablement, par le tableau idyllique qui leur avait été dépeint d'une contrée fertile et douce à vivre.

En 1717 et 1721, tout a été mis en œuvre pour donner une nouvelle image de la Louisiane. Cette propagande eut des effets immédiats et à long terme. ${ }^{42}$ Dès 1717, le Nouveau Mercure publie une "Dissertation particulière sur la Louisiane" dont les descriptions se retrouveront dans d'autres mémoires et articles. Dans la région des Houmas "commencent les bonnes terres... il n'y a qu'à y mettre la charrüe et ensuite semer". La région est très fertile: "tous les grains d'Europe viendront (remarquez le futur!) à merveille dans ces terres vierges, excepté les bords de la mer... dans les jardins dont on a eu soin, tous les légumes de France, les Pèches, les Arbricotiers (sic), les Poiriers, les pomiers, les muscats, etc y ont fort bien réussi". ${ }^{33}$ Par la suite, le ton devient dithyrambique. En mai 1719, le Nouveau Mercure publie une lettre qu'un nouvel arrivé écrit à sa femme: "Je suis actuellement, dit-il, dans les dépendances de la ville, plus en sureté que dans une citadelle, quoiqu'entouré de Sauvages et de Français, avec lesquels on ne court aucun risque. La bonne foi est telle que chacun laisse ses portes ouvertes, s'entr'aidant les uns les autres." Les mœurs simples et honnêtes rappellent celles d'un âge d'or, car dans ce pays "on ... préfère le commerce, la culture des terres, des arbres et des plantes à toutes les choses vaines et inutiles". Dans ce paradis pastoral, la chère est abondante: "La terre y est excellente et d'un grand rapport. Elle

42 Voir Pierre H. Boulle, "Some Eighteenth-Century Views of Louisiana," in Frenchmen and French Ways in the Mississippi Valley, édité par John Francis McDermott (Urbana, 1969), 15-27. (1719) : 92, 83. 
produit toutes sortes de légumes et de fruits, beaucoup meilleurs qu'en France, et en bien plus grand nombre. L'on a à fort bas prix des vaches, cochons, poulets, etc. Le pays est fertile en bœufs sauvages, chevaux, ours, léopards, reptiles et autres animaux qui fuient devant les hommes et ne leur font aucun mal. Tous les gibiers de France, et d'autres qui n'y sont pas connus, y abondent, ils sont de très bon goût. Les sauvages en fournissent tant que l'on veut pour très peu de chose". ${ }^{44}$ On accompagne cette description idyllique du Nouveau Monde d'estampes qui représentent les Indiennes préparant de la soie ou assemblant des émeraudes, et surtout d'aimables indigènes accueillant les Français "avec des témoignages de respect et d'admiration".45

En réalité, la Nouvelle-Orléans n'est encore en 1720 qu'un cantonnement de "deux cents personnes qu'on a envoyées pour bâtir une ville et qui sont campées au bord d'un grand fleuve; or elles ne songent qu'à se mettre à couvert des injures de l'an, en attendant... qu'elles ayent bati des maisons". ${ }^{46}$ Mais le correspondant du Nouveau Mercure parle des "maisons ... simples, basses comme dans nos campagnes, couvertes de grandes écorces d'arbres et de grosses cannes". ${ }^{47}$

Dans ces descriptions de la Louisiane on retrouve toujours les mêmes leitmotive: la terre est si fertile que les récoltes viennent spontanément; les vignes sauvages portent des raisins délicieux dont on peut tirer un excellent vin; le gibier abonde et les récoltes viennent deux fois par an. Quiconque connaît tant soit peu la géographie classique et médiévale reconnaît sans peine le motif des Iles Fortunées, et le paradis terrestre des géographes, de Pline l'Ancien à Pierre d'Ailly, qui se situe toujours au-delà du monde connu. Ce paradis terrestre, en l'occurrence, se trouvait sur les rives du Mississipi, et la Compagnie des Indes offrait d'y transporter gratuitement les immigrants.

Malheureusement le motif des Iles Fortunées porte toujours son corollaire: fruits et récoltes poussent sans besoin d'effort humain, et il semble bien que ce soit là ce que les colons attendaient, consciemment ou non, du sol louisianais. Il est donc peu étonnant qu'ils aient été fort déçus, et encore moins surprenant que, pour un pays où les récoltes venaient si aisément, l'on n'ait pas cherché à recruter de véritables paysans. Bienville s'était toujours amèrement plaint des habitants - sans pousser toutefois

44 Nouveau Mercure (mai 1719) : 185-186.

45 Gravier, 34.

46 Charlevoix, tome VI, lettre XXXII: 208.

47 Nouveau Mercure (mai 1719) : 185. 
aussi loin que Cadillac qui jugeait la population de "lie du Canada, gens de sac et de corde, sans respect pour la religion".48 Bienville traitait les soldats sous ses ordres de "déserteurs, fauconniers, et coquins" ${ }^{49}$, et, selon lui, les habitants ne valaient guère mieux. En 1759 Kerlérec se plaignait encore de la fainéantise des colons louisianais, sauf des familles alsaciennes récemment arrivées. On comprend sans peine l'empressement avec lequel Kerlérec reçut ces nouveaux immigrants, les paysans étant toujours rares en Louisiane malgré l'essor démographique remarquable de la période de Law.

Cette période de colonisation intensive a fait l'objet de nombreuses critiques. On a reproché à Law d'avoir envoyé sur les rives louisianaises des immigrants inutiles qu'il fallut nourrir. Il est indubitable que l'émigration forcée, accueillie avec enthousiasme en 1717 pour résoudre le problème de peuplement, ne donna que de médiocres résultats. Mais la propagande de Law et l'intérêt très réel que, pour la première fois, l'on manifesta pour les possibilités commerciales de la colonie encouragèrent une immigration qui fut à l'origine de la véritable colonisation de la Louisiane. Après Law, il n'y eut plus d'effort de peuplement jusqu'au milieu du XVIII ${ }^{e}$ siècle alors que l'on assista à un mouvement d'immigration alsacienne que la Guerre de Sept Ans devait malheureusement interrompre à ses débuts. 